\title{
Aclacinomycin Antitumor Antibiotic
}

National Cancer Institute

\section{Source}

National Cancer Institute. Aclacinomycin Antitumor Antibiotic. NCI Thesaurus. Code C987.

An oligosaccharide anthracycline antibiotic isolated from strains of the bacterium

Streptomyces galilaeus with potential antineoplastic activity. These agents display potent antitumor properties due to their ability to intercalate into DNA and interfere with the function of both the topoisomerase I and II enzymes, thereby inhibiting DNA replication and ultimately, interfering with RNA and protein synthesis. 